\title{
Study of solvent effect in laser emission from Coumarin 540 dye solution
}

\author{
Ritty J. Nedumpara, ${ }^{1, \star}$ Thomas K. J., ${ }^{1}$ Jayasree V. K., ${ }^{1}$ C. P. Girijavallabhan, ${ }^{2}$ V. P. N. Nampoori, ${ }^{1}$ \\ and P. Radhakrishnan ${ }^{1}$ \\ 'International School of Photonics, Cochin University of Science and Technology, Cochin, 682 022, India \\ ${ }^{2}$ Centre of Excellence in Lasers and Optoelectronic Sciences, Cochin University of Science and Technology, \\ Cochin, 682 022, India \\ *Corresponding author: rittynedumpara@yahoo.com
}

Received 27 November 2006; revised 12 March 2007; accepted 21 March 2007; posted 23 March 2007 (Doc. ID 77412); published 6 July 2007

\begin{abstract}
The results of a brief investigation of the amplified spontaneous emission and lasing characteristics of Coumarin 540 dye in as many as ten different solvents are reported. It has been found that C 540 dye solutions contained within a rectangular quartz cuvette give laser emission with well resolved equally spaced modes when pumped with a $476 \mathrm{~nm}$ beam. The modes were found to originate from the subcavities formed by the plane-parallel walls of the cuvette containing the high-gain medium. While the quantum yield remains a decisive factor, a clear correlation between the total width of the emission spectra and the refractive indices of the solvents of the respective samples has been demonstrated. The well-resolved mode structure exhibited by the emission spectra gives clear evidence of the lasing action taking place in the gain medium, and the number of modes enables us to compare the gain of the media in different samples. A detailed discussion of the solvent effect in the lasing characteristics of C540 in different solutions is given. 2007 Optical Society of America
\end{abstract}

OCIS codes: $140.2050,140.3380$.

\section{Introduction}

The wide use of organic dyes and dye doped polymers in optical and optoelectronic applications has produced a renewed interest in the area of laser dres [1-5]. The broad spectral range covering the blue to the IR region, the high efficiency, the large spectral bandwidth, and fairly simple implementation offer unique operational flexibility for dye lasers. Recently the coumarin dyes are drawing renewed interest for the development of blue and green lasers owing to their emission in this region of the waveiength. With their large quantum yields and strong absorption, the coumarin dyes are excellent laser gain media in the near-UV to green wavelength region [6-9]. Many coumarin derivatives are found to be strongly fluorescent with their quantum yield oten close to unity [10]. Coumarin-doped biphenyl films are suggested to have possible applications as

0003-6935/07/214786-07\$15.00/0

Q 2007 Optical Society of America organic semiconductors, efficient laser media, and optical memory materials. Recently coumarin dyes have been introduced as dopants in electrically excited organic light emitting diodes (OLED). They have also been successfully introduced in high-brightness OLED devices $[11,12]$.

The coumarin dyes are found to be very sensitive to the polarity of solvents and to microenvironments. Strong polarity-dependent Stokes's shifts are observed in many coumarin derivatives [11-16]. The 7-NEt $\mathrm{N}_{2}$ coumarin dyes are seen to exhibit quite unusual behavior in certain nonpolar solvents. It is reported that the dye adopts different conformations of this group in nonpolar and some other solvents [1113]. A linear relation is reported between quantum yield and solvent polarity for moderate to high polarity solvents but it deviates to higher values in nonpolar solvents [16].

Here we report the effect of solvents on certain characteristics of laser emission observed from Coumarin 540 (C 540) dye solutions. Since the C 540 dye is a high-gain medium, for a pump intensity of 
$65 \mathrm{~kW} / \mathrm{cm}^{2}$, laser emission is observed with well resolved, equally spaced resonant modes. The mode structure in the emission spectrum is attributed to laser emission due to the optical feedback from the parallel windows of the quartz cuvette in which the dye solutions are taken. A more or less linear relationship is observed between the quantum yield and the laser modes in most of the solvents used, with exceptions being seen for a few highly polar solvents. For such solvents the refractive index of the solvent is found to be a decisive factor, which moderates the lasing efficiency of the dye. We have done the investigations in a variety (ten) of solvents, which comprises polar protic, dipolar aprotic, and nonpolar solvents.

\section{Experimental Details}

The gain medium for the present study was C 540 dye in different solvents. Coumarin dye shows excellent solubility in all these solvents. Figure 1 shows the typical absorption spectra of dye in ethanol and toluene with polarity indices 5.2 and 2.4 , respectively. These spectra were recorded using a UV-VIS spectrophotometer (JascoV-570, Japan). The dye solution in ethanol with a concentration of $1 \times 10^{-6} \mathrm{M}$ has the absorption peak at $456 \mathrm{~nm}$ with a bandwidth of $74 \mathrm{~nm}$, whereas the peak is at $442 \mathrm{~nm}$ with a bandwidth of $64 \mathrm{~nm}$ for $\mathrm{C} 540$ in toluene.

The first part of the experiment was conducted in dye dissolved in methanol. The concentration of choice was $4 \times 10^{-4} \mathrm{M}$. The laser emission studies of the dye solution were made by taking the sample solution in a quartz cuvette of $1 \mathrm{~cm}$ inner length. The emission spectra were recorded by exciting the sample with $476 \mathrm{~nm}$ radiation from a Quanta Ray MOPO (MOPO 700, USA), pumped by $Q$-switched Nd:YAG laser at $355 \mathrm{~nm}$ that emits pulses of $7 \mathrm{~ns}$ duration at a repetition rate of $10 \mathrm{~Hz}$. A cylindrical lens was used to focus the pump beam in the shape of a stripe on the

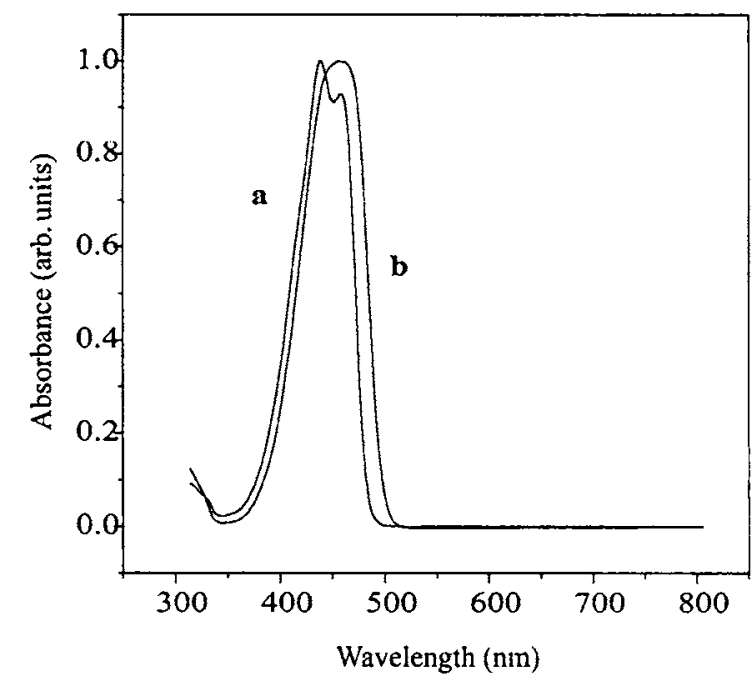

Fig. 1. Absorption spectra of C 540 solution in (a) toulene, (b) ethanol. sample. Owing to the high-absorption cross section of the dye solution, the pump beam was fully absorbed by the front layer of the sample, and it created a stripe like an excited gain medium. A vertical slit was incorporated in the path of the beam between the cylindrical lens and the sample so as to vary the stripe length on the sample, and it was adjusted to fix the pump beam width of $7 \mathrm{~mm}$ in the present case. The output was collected from the edge of the front surface of the cuvette using an optical fiber in a direction normal to the pump beam. The emission spectra were recorded with an Acton monochromator attached with a CCD camera. The emitted beam from the edge of the cuvette was so strong and highly directional that we could collect it without any focusing.

In the second part of the investigations, the emission spectra were recorded for ten solvents of different polarities to study the effect of solvents on laser emission from the dye solution. All the investigations were done in dye solutions with a constant concen. tration of $4 \times 10^{-4} \mathrm{M}$, while all other experimental conditions such as pump intensity, excitation length of the gain medium, and mode of collection remained the same.

\section{Results and Discussions}

To study the nature of emission from the dye dissolved in methanol, the emission spectra were recorded for various pump intensities starting from $45 \mathrm{~kW} / \mathrm{cm}^{2}$, keeping the excitation length of the beam as $7 \mathrm{~mm}$. For a pump beam intensity of $45 \mathrm{~kW} / \mathrm{cm}^{2}$, the fluorescence spectrum recorded was highly broad with a spectral width of $33 \mathrm{~nm}$. With the increase in pump intensity, the fluorescence emission spectrum narrowed and gave amplified spontaneous emission (ASE). With a pump intensity of $65 \mathrm{~kW} / \mathrm{cm}^{2}$, ASE was observed with a spectral width of $7 \mathrm{~nm}$ (Fig. 2). When the pump intensity was further increased, a periodic modulation structure was observed in the

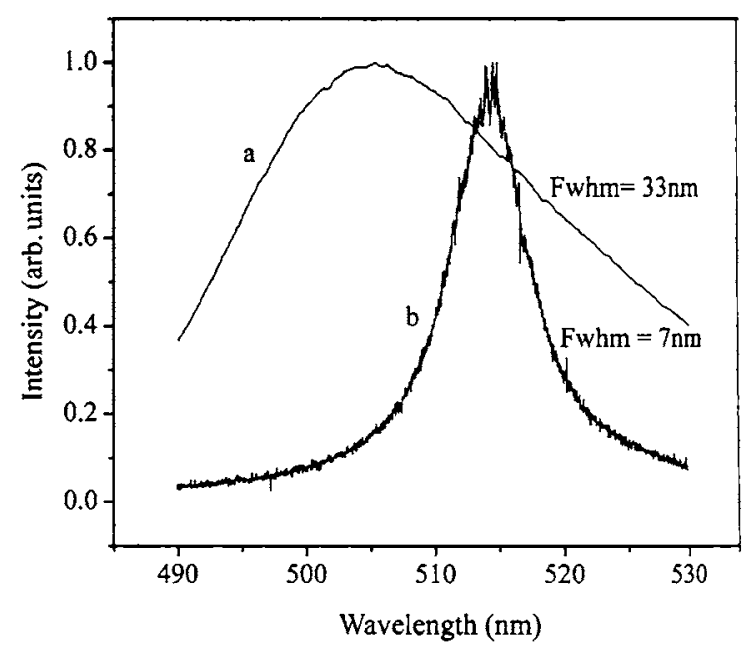

Fig. 2. Emission spectra of $\mathrm{C} 540$ solution in methanol (a) fluorescence spectrum, (b) ASE. 


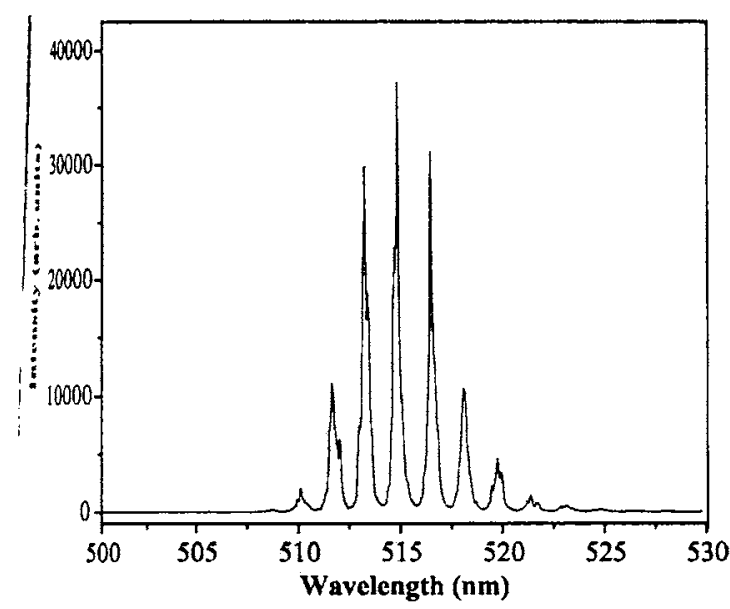

jig. 3. Lasing spectrum of C 540 solution in methanol. Resonant sdes with spacing $1.62 \mathrm{~nm}$.

mission spectrum. At higher pump intensities, well resolved equally spaced resonant modes were observed, which could be attributed to laser emission where the emission beam was highly directional (Fig. 3). The occurrence of similar resonant modes was reported by Yokoyama et al. [17] in a dye medium added with dendrimers. By encapsulating the dye, the dendrimer reduced the self-aggregation and the molecular quenching of the dye at higher concentratons and thereby increased its gain. They had related the spacing of the modes to the penetration depth of the beam in the medium. To verify the validity of this explanation, the emission spectra were recorded for various concentrations of the dye ranging from $1 \times 10^{-4}$ to $8 \times 10^{-4} \mathrm{M}$, which corresponds to different penetration depths. The same mode structure was repeated with more or less equal spacing and a redshift in the spectra (Fig. 4). This confims that the mode spacing is not directly related to penetration depth.

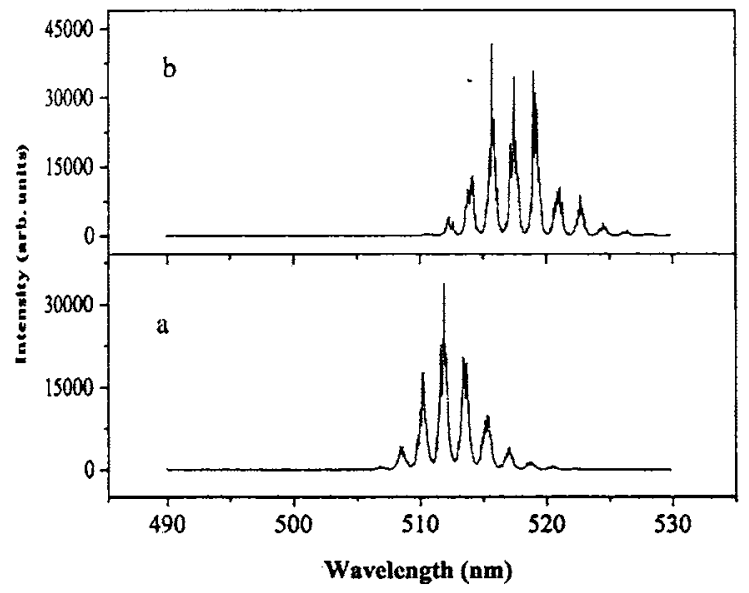

Fig. 4. Lasing spectra in methanol for two concentrations. (a) $1 \times 10^{-4} \mathrm{M}$, (b) $8 \times 10^{-4} \mathrm{M}$.
Further investigations were done by changing the excitation length of the pump beam. For a dye solution of concentration $4 \times 10^{-4} \mathrm{M}$ and pump beam intensity above $150 \mathrm{~kW} / \mathrm{cm}^{2}$, the emission spectra showed the features of ASE when the excitation length was increased to $2 \mathrm{~mm}$. The spectral width was again approximately $7 \mathrm{~nm}$ for this length, but when the excitation length was further increased the narrowed spectrum showed well resolved resonant peaks with equal spacing. With a further increase in excitation length, the modes became prominent with very high output intensity. The emission spectrum observed in the dye, dissolved in methanol for an excitation length of $4 \mathrm{~mm}$, was exactly similar to the one observed for an excitation length of $7 \mathrm{~mm}$ (Fig. 3). An increase in pump intensity gave the same mode structure with the same mode spacing. However, the less prominent modes increased in their strength at higher pump intensities.

The occurrence of mode structure from dye solution was reported by Guang et al. [18] where the laser emission was attributed to the Fresnel reflection feedback from the two parallel optical windows of the cuvette. Considering the two surfaces of the windows and the gain medium, a multicavity structure can be formed between the two plane-parallel walls of the cuvette. The resonant conditions for the four subcavities formed between the two windows can be written as

$$
\begin{aligned}
2\left[n L+n^{\prime}\left(l_{1}+l_{2}\right)\right] & =K_{1} \lambda, \\
2 n L & =K_{2} \lambda, \\
2\left(n L+n^{\prime} l_{2}\right) & =K_{3} \lambda, \\
2\left(n L+n^{\prime} l_{1}\right) & =K_{4} \lambda,
\end{aligned}
$$

where $L$ is the length of the dye medium, $n^{\prime}$ is the refractive index of quartz, $l_{1}$ and $l_{2}$ are the thicknesses of the two parallel walls of the cuvette, $n$ is the refractive index of the gain medium and $K_{1}, K_{2}, K_{3}$, and $K_{4}$ are arbitrary integers [18]. The resonance condition providing the widest spectral periodicity is obtained by the subtraction of the last two conditions of Eq. (1), which yields

$$
2 n^{\prime}\left(l_{2}-l_{1}\right)=K \lambda \text {, }
$$

where $K=\left(K_{3}-K_{4}\right)$.

This equation is equivalent to the maximum transmission condition of a Fabry-Perot etalon. Thus the partial reflections from the windows of the cuvette produce the effect of a Fabry-Perot etalon and provide the optical feedback necessary for laser emission. For the lasing spectrum the wavelength spacing between the different modes is given by

$$
\Delta \lambda=\frac{\lambda^{2}}{2 n^{\prime}\left(l_{2}-l_{1}\right)},
$$




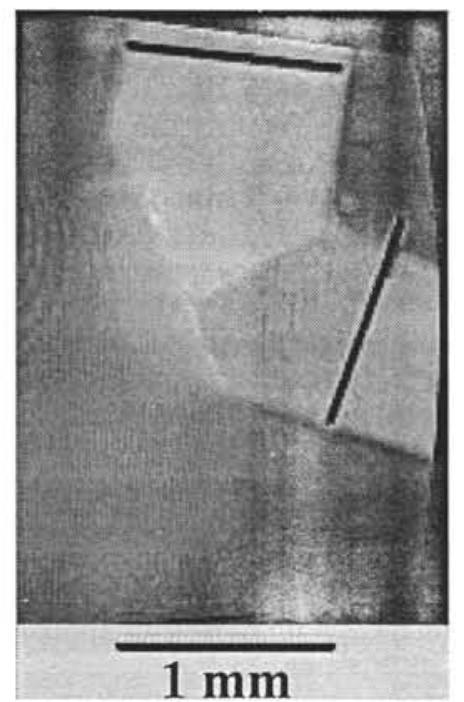

Fig. 5. Typical figure showing the variations in the thickness of the bottom and one of the side walls of a cuvette of $1 \mathrm{~cm}$ thickness (photograph of the image obtained with microscope attached with a CCD camera).

where $\lambda$ is the average lasing wavelength. Substituting for refractive index $n^{\prime}$ as 1.46 , the average lasing wavelength $\lambda$ as $515 \mathrm{~nm}$, and the mode spacing $\Delta \lambda$ as $1.62 \mathrm{~nm}$ from the experiment with a cuvette of $1 \mathrm{~cm}$ path length, $l_{2}-l_{1}$ is estimated as $56 \mu \mathrm{m}$. To check the validity of the above conclusion, the thickness of the windows was accurately measured using a microscope attached with a CCD camera (Panasonic) and the $l_{2}-l_{1}$ value was found to be $55 \pm 5 \mu \mathrm{m}$ at different positions of the cuvette, which is in close agreement with the observed value. The photograph of the CCD image taken for two walls of a cuvette is given in Fig. 5, which clearly indicates the change in thickness of the different walls of the cuvette. To confirm this result, the emission spectrum of the same dye solution in a cuvette of path length $0.5 \mathrm{~cm}$ was taken. Figure 6 shows the mode structure observed with a different spacing of $0.92 \mathrm{~nm}$. Substituting for the peak emission as $514 \mathrm{~nm}$, the thickness difference $\left(l_{2}-l_{1}\right)$ of the parallel walls was obtained as $98 \mu \mathrm{m}$. The measured value of $\left(l_{2}-l_{1}\right)$ for this $0.5 \mathrm{~cm}$ cuvette was $100 \pm 5 \mu \mathrm{m}$, which confirms the existence of a Fabry-Perot optical cavity effect due to the reflections from the walls of the cuvette.

The incidence angle of the pump beam was varied in order to check the effect of its deviation from the cavity axis on the mode structure. We could observe the mode structure even when the cuvette was tilted to approximately $15^{\circ}$ from the direction of incidence $[17,18]$. Owing to the high gain of the dye medium, even a portion of the reflection from the windows along the cavity axis is sufficient to produce the cavity lasing.

In the second part of the study, the features of the mode structure were employed to investigate the effect of solvents on the laser emission of the dye. De-

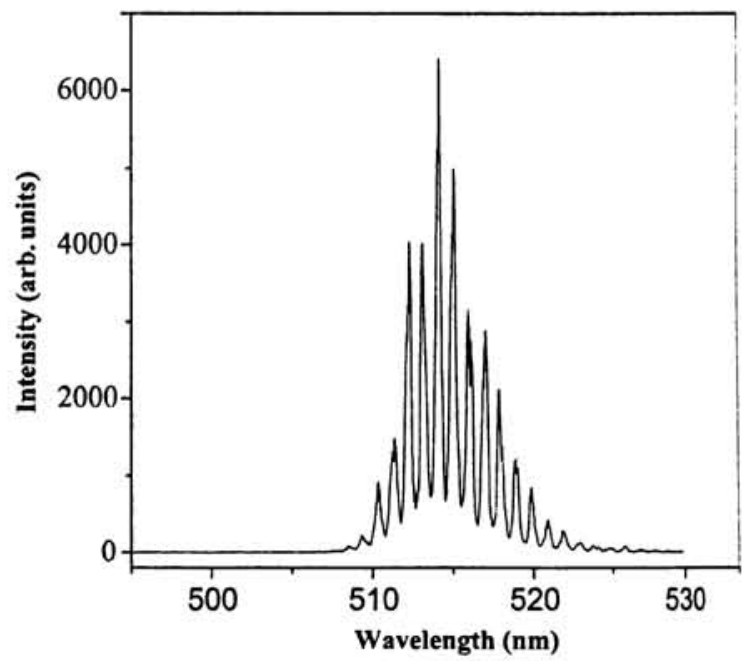

Fig. 6. Laser emission spectrum of dye solution in a cuvette of $0.5 \mathrm{~cm}$. Mode spacing $0.92 \mathrm{~nm}$.

tailed investigations were done with ten solvents of different quantum yields $\left(\Phi_{f}\right)$ and polarities. The photophysical properties of laser dyes were found to be highly sensitive to the nature of solvents and to the environments $[11,14-16]$. The polarity of solvents, their viscosity, and dielectric constants are found to be critical in the photochemical behavior of laser dyes. The solvents of choice include polar protic, di. polar aprotic and nonpolar ones. A comparative method [19] was applied to estimate the quantum yield values in different solvents using Rhodamine 6G $(\mathrm{Rh} 6 \mathrm{G})$ in ethanol as the reference [20]. Absorp. tion and fluorescence spectra were recorded using a Jasco, Japan, V-532 UV-VIS spectrophotometer and a Cary Eclipse, Australia, fluorescence spectrophotometer, respectively. Both polar protic and dipolar aprotic solvents exhibited very high quantum yeld. Coumarin dye in ethanol has a quantum yield of approximately 1 , and toluene, which is a nonpolar solvent, gives the least value of 0.76 . Though a direct correlation between $\Phi_{f}$ and polarity could not be observed, generally the $\Phi_{f}$ values were found to be high with solvents of high polarity.

The emission spectra were recorded for all the sol. vents with a fixed dye concentration of $4 \times 10^{-4} \mathrm{M}$. All the experimental conditions remained the same throughout the investigations. Interesting results were obtained and these showed a correlation between the quantum yield of the dye in different sol. vents and the mode structure observed due to cavity lasing. With ethanol, which has a quantum yield of $\sim 1$, the emission spectrum recorded has a total spec. tral width of $7 \mathrm{~nm}$ consisting of approximately 12 modes (Fig. 7). With methyl ethyl ketone having a quantum yield of 0.97 , the spectral width was $5.6 \mathrm{~nm}$ with nine as the number of modes (Fig. 8). For butyl acetate with a quantum yield of 0.85 , the number of modes was reduced to five (Fig. 9). In the case of toluene with a quantum yield 0.76 , no mode pattern 


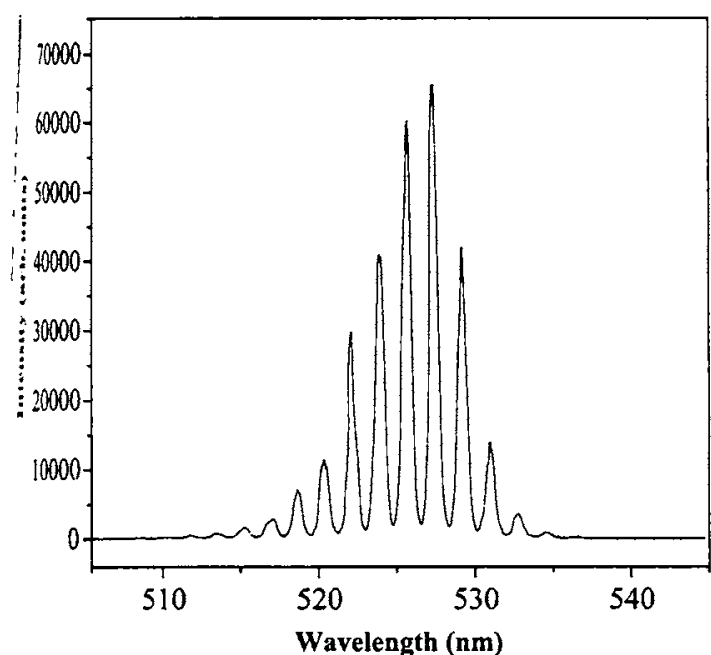

7. 7. Laser emission spectrum from C 540 dye solution in ethnol. Mode spacing $1.65 \mathrm{~nm}$.

zas observed. Toluene gives only an ASE spectrum ith a total spectral width of $3.4 \mathrm{~nm}$ (Fig. 10). The fye medium is found to be sensitive to the polarity of the solvent in its nature of laser emission. In general, the gain of the medium increases with an increase in polarity. But the laser emission is observed to be high in thanol, which is less polar compared to methanol. This may be attributable to the more viscous nature fethanol, which may reduce the molecular interacton between dye and solvent molecules. Table 1 gives a comparison of solvent polarity, refractive index, quantum yield, and the number of modes observed in the emission spectrum.

Though Table 1 shows the correlation between the number of modes and the quantum yield of the dye in different solvents, some discrepancy is noticeable in the case of a few high-polarity solvents. In the case of dimethyl formamide (DMF), the number of modes is

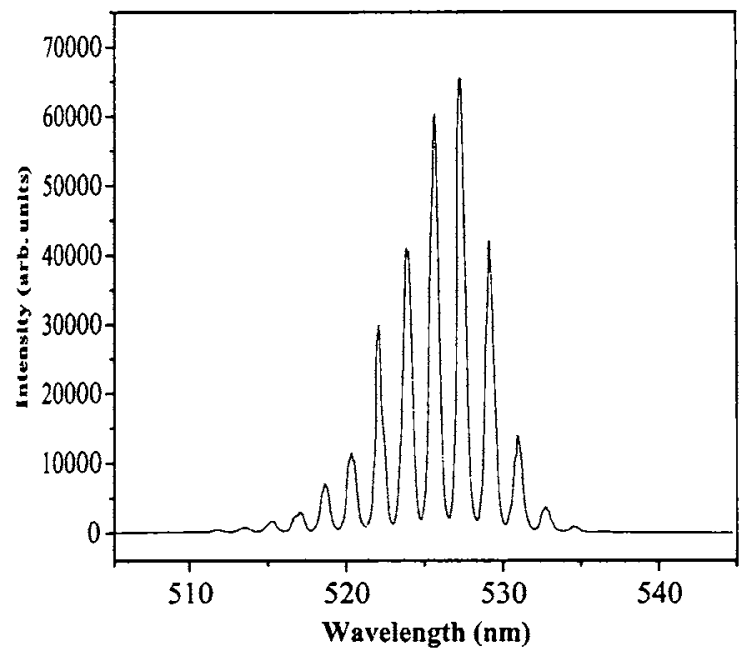

Fig. 8. Laser emission spectrum from C 540 dye solution in MEK. Mode spacing $1.75 \mathrm{~nm}$.

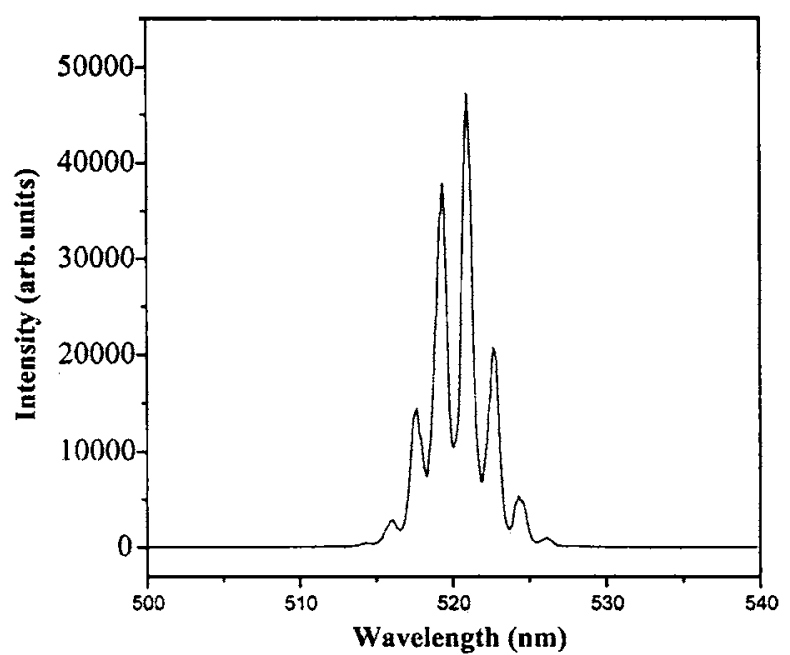

Fig. 9. Laser emission spectrum of dye solution in butyl acetate. Mode spacing $1.69 \mathrm{~nm}$.

fewer and for dimethyl sulphoxide (DMSO) no laser emission is observed though both of them have high quantum yields. Cyclohexanone which is a less polar solvent, also shows the same result. All these solvents have a comparatively high refractive index, which is close to the refractive index of quartz. The reflection coefficient of the surfaces is also an important parameter in determining the lasing threshold. For a gain medium in a resonator cavity with length $L$ and endface reflectivities $R_{1}$ and $R_{2}$, oscillations will build up when the loop gain $R_{1} R_{2} \exp (2 g L)>1$ [21]. The reflectivity at the end face is given by

$$
R=\left(\frac{n_{1}-n_{2}}{n_{1}+n_{2}}\right)^{2}
$$

The absence of lasing modes in the above solvents can be attributed to the reduced value of the reflection

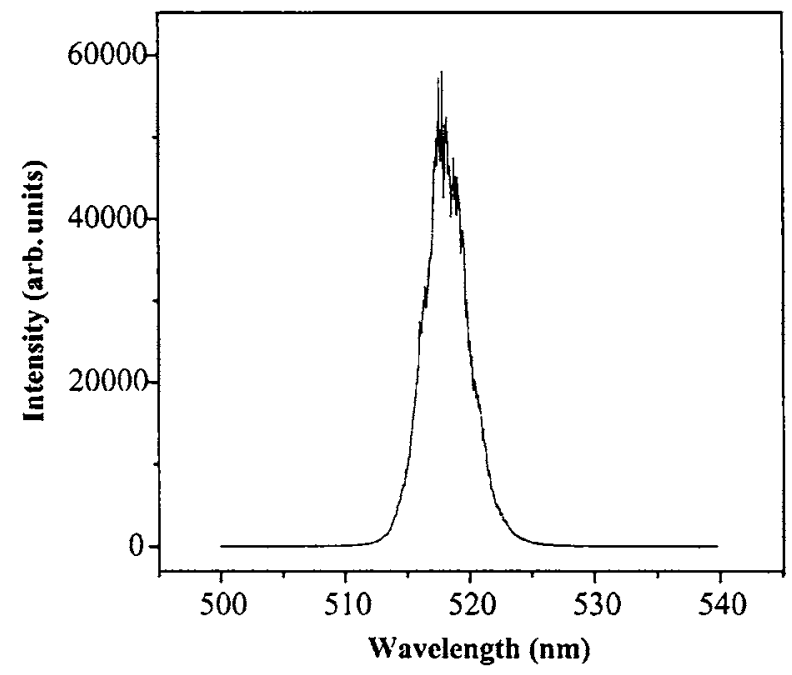

Fig. 10. ASE spectrum of dye solution in toluene. 
Table 1. Comparison of Polarity, Quantum Vield, Refractive Index, Number of Modes and Reflectivity for Different Solvents

\begin{tabular}{|c|c|c|c|c|c|c|}
\hline Solvent & Nature & Polarity Index & Ref. Index & Q. Yield & No. of Modes & $R \times 10^{-5}$ \\
\hline Ethanol & $\mathrm{pp}^{\alpha}$ & 5.2 & 1.36 & 0.99 & 12 & 125.7 \\
\hline Dichloromethane & $\operatorname{dap}^{b}$ & 3.4 & 1.42 & 0.98 & 10 & 19.29 \\
\hline Methyl ethyl ketone & dap & 4.5 & 1.376 & 0.97 & 9 & 87.7 \\
\hline Methanol & $\mathrm{pp}$ & 6.6 & 1.326 & 0.91 & 7 & 231.3 \\
\hline Butyl acetate & dap & 3.9 & 1.394 & 0.85 & 5 & 53.4 \\
\hline Dimethyl formamide (DMF) & dap & 6.4 & 1.431 & 0.94 & 4 & 10.06 \\
\hline Dioxane & dap & 4.8 & 1.42 & 0.8 & 3 & 19.29 \\
\hline Toulene & $\mathrm{np}^{c}$ & 2.4 & 1.494 & 0.76 & - & 13.24 \\
\hline Dimethyl sulphoxide (DMSO) & dap & 6.5 & 1.478 & 0.96 & - & 3.75 \\
\hline Cyclohexanone & dap & 4.5 & 1.45 & 0.97 & 一 & 1.18 \\
\hline
\end{tabular}

${ }^{a}$ Polar protic.

${ }^{b}$ Dipolar aprotic.

'Nonpolar.

coefficients. For example, cyclohexanone with a refractive index of 1.45 acts as an index matching medium yielding a negligible reflection coefficient $\left(R=1.181 \times 10^{-5}\right)$ and laser emission is not observed with such a small feedback, resulting from the negligible value of $R$. In the case of ethanol, where the difference in refractive indices is comparatively large giving $R=125.7 \times 10^{-5}$, a good lasing spectrum is obtained at a low threshold pump beam intensity. For comparison, the reflectivity at the end faces for different solvent media is also included in Table 1 . The lasing behavior of the gain medium is explicit from the nature of the emission spectrum observed with the mode structure. The total spectral width of the emission spectra gives a clear indication of the gain of the medium in different solvent environments. A correlation is explicit between the gain of the lasing medium and the spectral width of emission and correspondingly the mode structure exhibited by it.

The emission spectrum of $\mathrm{Rh} 6 \mathrm{G}$, the best known of all laser dyes is also recorded for the same absorbance as coumarin dye in methanol. The emission spectrum

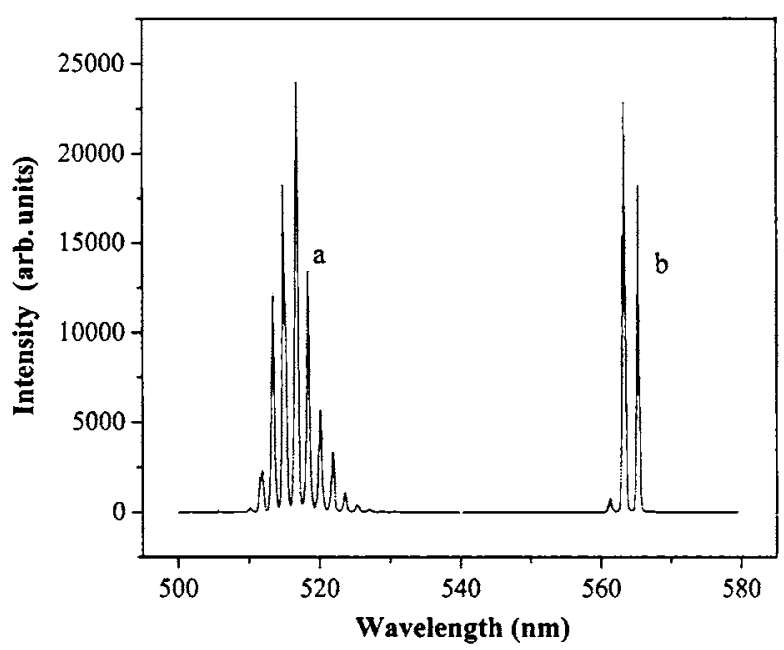

Fig. 11. Emission spectrum in methanol (a) C 540, mode spacing $1.65 \mathrm{~nm}$, (b) Rh $6 \mathrm{G}$, mode spacing $2.01 \mathrm{~nm}$. exhibited only two modes with a spectral width of $2.4 \mathrm{~nm}$ whereas the emission spectrum of coumarin has a spectral width of $5 \mathrm{~nm}$ with seven to eight modes (Fig. 11). Even though the quantum yield of $C$ 540 in methanol is slightly lower than that of $\mathrm{Rh} 6 \mathrm{G}$, the spectral width for C 540 is found to be greater than that of $\mathrm{Rh} 6 \mathrm{G}$, which is the well-known efficient laser dye.

\section{Conclusion}

The gain characteristics of C 540 in different solvents are investigated. Laser emission is observed from dye solution taken in a quartz cuvette. The partial reflections from the two parallel walls of the cuvette provided the optical feedback necessary for laser emission. The subcavities formed between the windows of the cuvette produced the effect of a FabryPerot optical cavity resulting in well-resolved equally spaced resonant modes. The mode spacing was related to the difference in thickness of the paralle! walls of the cuvette.

The features of the mode structure in the emission spectra are employed to study the effects of solvents on the laser emission of the dye. Dye solutions with high quantum yields exhibited good lasing spectra with a finite number of modes. The discrepancy noticed in certain solvents of high quantum yield, with a reduced number of modes or no lasing at all, is explained in terms of the low reflection coefficient. These solvents with refractive indices close to the refractive index of quartz act as index matching media yielding a low reflection coefficient, which is insufficient to produce the necessary feedback for laser emission. The observation of mode structure is a clear evidence of laser emission from C 540 dye solution. The total width of the emission spectrum observed for the gain medium in different solvent environments clearly marks the gain of the medium. The emission spectra with sufficient gain exhibited laser modes. The gain, in turn, is a function of quantum yield and the refractive index of the medium. This work enables us to make a comparison of the gain of the media and the spectral width of the emission spectra exhibited by them in different solvent environments. 
R J. Nedumpara thanks the University Grants immission, New Delhi, India for financial support the form of an FIP grant. Thomas K. J. is grateful athe Council of Scientific and Industrial Research, lee Delhi, for the research fellowship. The assisanse from the UGC under the UPE program is also atrowledged.

\section{terences}

i. A. Costela, I. Garcia-Moreno, and R. Sastre, "Polymeric solidstate dye lasers: recent developments," Phys. Chem. Chem. Phys. 5, 4745-4763 (2003).

2. M. A. Diaz-Garcia, S. F. De Avila, and M. G. Kuzyk, "Dye doped polymers for blue organic dyes," Appl. Phys. Lett. 80, 44864888 (2002)

3. B. F. Howell and M. G. Kuzk, "Lasing action and photodegradation of Disperse Orange 11 dye in liquid solution," Appl. Phys. Lett. 85, 1901-1903 (2004).

4. Otomo, S. Yokoyama, T. Nakahama, and S. Mashiko, "Supernarrowing mirrorless laser emission in dendrimer-doped polymer waveguides," Appl. Phys, Lett. 77, 3881-3883 (2000).

5. W. Lu, B. Zhong, and D. Ma, "Amplified spontaneous emission and gain from optically pumped films of dye doped polymers," Appl. Opt. 43, 5074-5078 (2004).

6. F. J. Duarte, L. S. Liao, and K. M. Vaeth, "Coherence characteristics of electrically excited tandem organic light-emitting diodes," Opt. Lett. 30, 3072-3074 (2005).

7. S. S. Yap, W. O. Siew, T. Y. Tou, and S. W. Ng, “Red-green-blue loser emissions from dye-doped poly(vinyl alcohol) films, " Appl. Opt. 41, 1725-1728 (2002).

8. G. Gu, P. P. Ong, and Q. Li, "Photoluminescence of Coumarin 540 dye confined in mesoporous silica," J. Phys. D 32, 22872289 (1999).

9. F. J. Duarte, L. S. Liao, K. M. Vaeth, and A. M. Miller, "Widely tunable green laser emission using Coumarin 545 tetramethyl dye as the gain medium," J. Optics A 8, 172-174 (2006).

10. G. Jones II, W. R. Jackson, and C. Choi, "Solvent effects on emission yield and lifetime for coumarin laser dyes," J. Phys. Chem. 89, 294-300 (1985).

11. A. K. Satpati, M. Kumbhakar, D. K. Maity, and H. Pal, "Photophysical investigations of the solvent polarity effect on the properties of Coumarin-6 dye," Chem. Phys. Lett. 407, 114118 (2005).

12. A. Barik, S. Nath, and H. Pal, "Effect of solvent polarity on the photophysical properties of Coumarin-1 dye," J. Chem. Phys. 119, 10202-10208 (2003).

13. U. S. Raikar, C. G. Renuka, F. Nadaf, and B. G. Mulimani, "Solvent effects on the absorption and fluorescence spectra of Coumarin 6 and 7 molecules," Spectrochim. Acta Part A 65, 673-677 (2006).

14. H. Pal, S. Nad, and M. Kumbhakar, "Photophysical properties of Coumarin-120, unusual behavior in nonpolar solvents," J. Chem. Phys. 119, 443-452 (2003).

15. M. A. Haidekker, T. P. Brady, D. Lichlyter, and E. A. Theodorakis, "Effects of solvent polarity and solvent viscosity on the fluorescent properties of molecular rotors and related probes," Bioorg. Chem. 33, 415-425 (2005).

16. S. Nad and H. Pal, "Unusual photophysical properties of Coumarin-151," J. Phys. Chem. A 105, 1097-1106 (2001).

17. S. Yoloyama, A. Otomo, and S. Mashiko, "Laser emission from high-gain media of dye doped dendrimers," Appl. Phys. Lett. 80, 7-9 (2002).

18. S. Gaung He, R. Signorini, and P. N. Prasad, "Two-photon pumped frequency-upconverted blue lasing in coumarin dye solution," Appl. Opt. 37, 5720-5725 (1998)

19. A. T. R. Williams, S. A. Winfield, and J. N. Miller, "Relative fluorescence quantum yields using a computer-controlled luminescence spectrometer," Analyst (London) 108, 1067-1072 (1983).

20. D. Madgde, R. Wong, and P. G. Seybold, "Fluorescence quantum yields and their relation to lifetimes of Rhodamine $6 \mathrm{G}$ and Fluorescein in nine solvents," Photochem. Photobiol. 75, 327334 (2002).

21. W. Koechner, Solid-State Laser Engineering, Vol. 1 (SpringerVerlag, 1992). 